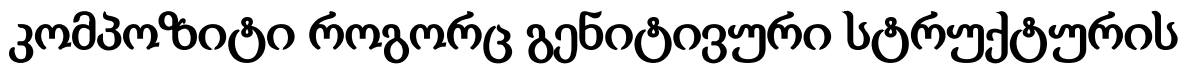 s oszobjäymgissto
}

\section{cosmo 6ojs 8 sdo}

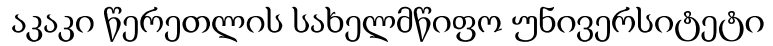

e-mail: dalichurzidse@yahoo.de

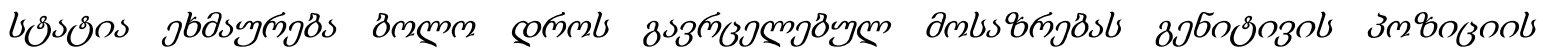

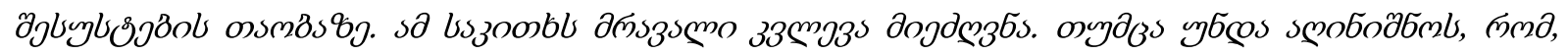

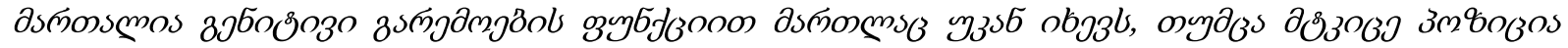

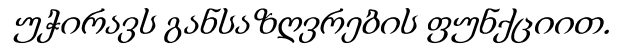

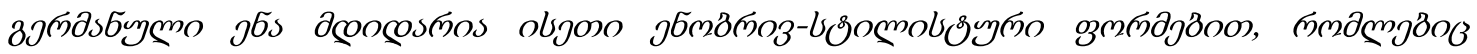

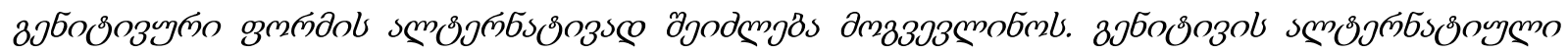

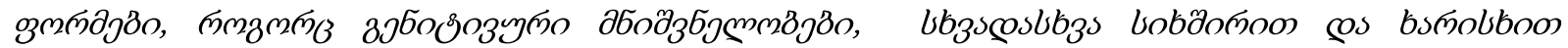

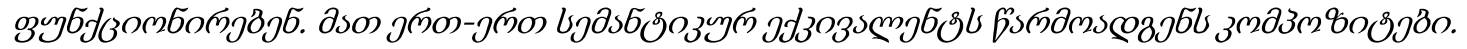

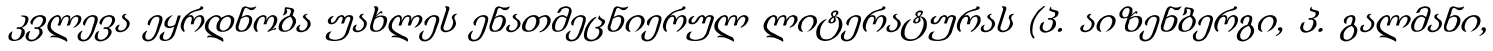

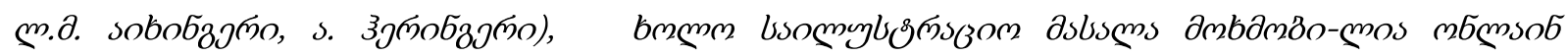

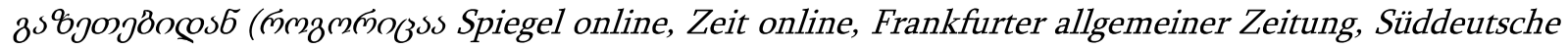

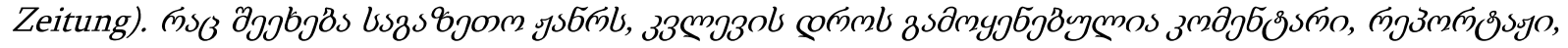

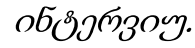

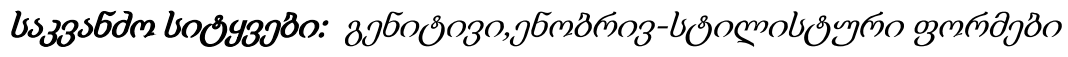




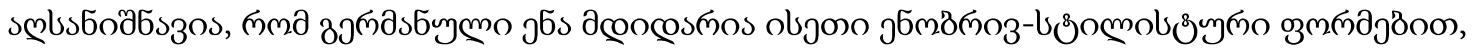

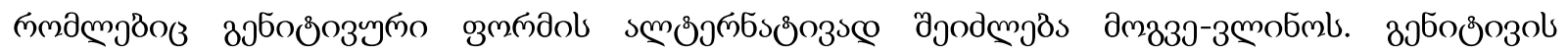

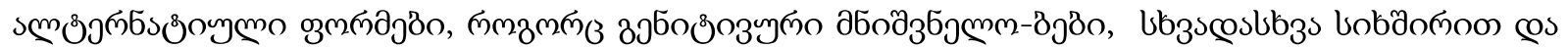

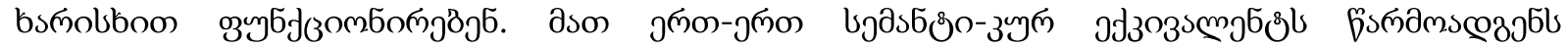

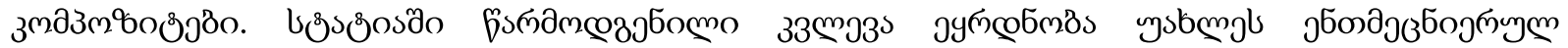

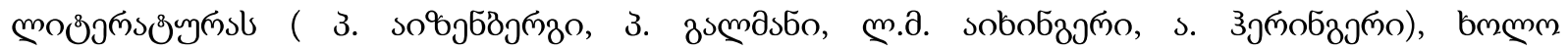

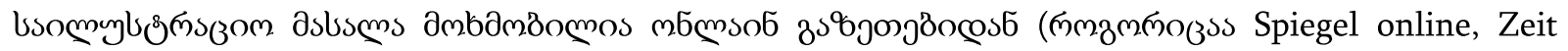

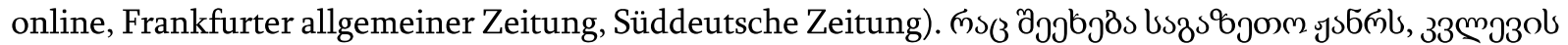

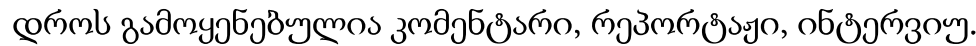

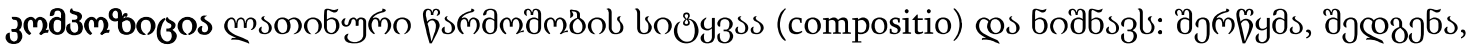

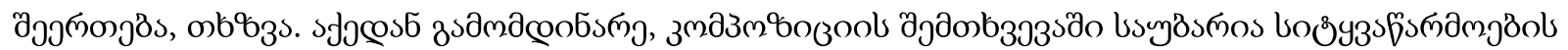

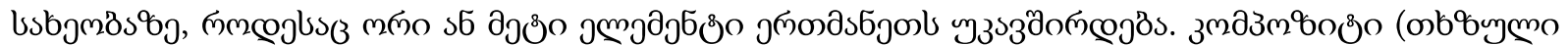

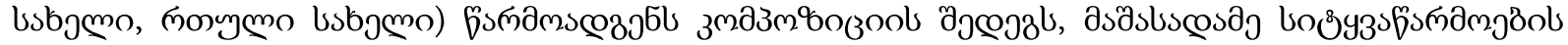

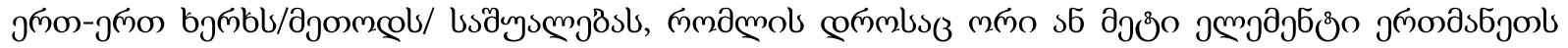

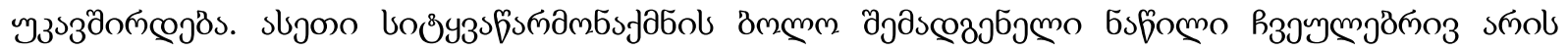

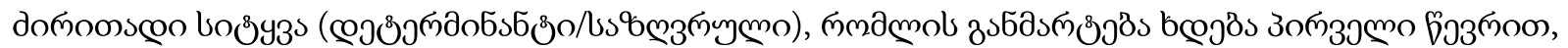

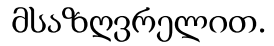

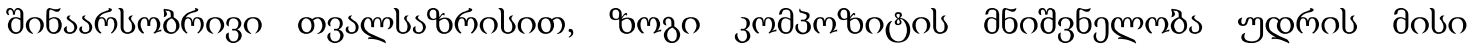

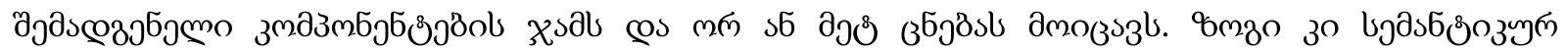

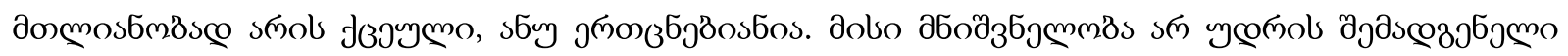

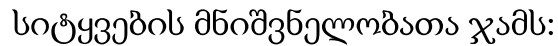

Allerdings wich Trump der Frage aus, ob er das Wahlergebnis als Verlierer anerkennen würde.[7]

Zuvor zeigte er dem feinen Washington seine Verachtung, indem er in kurzen, ausgebeulten Cargo-Hosen durch die Hauptstadt ging, unrasiert, zottelig.[4]

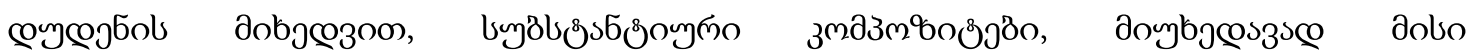

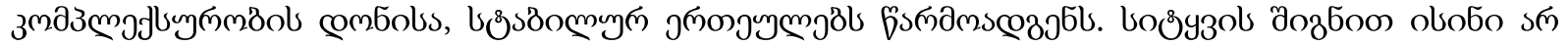

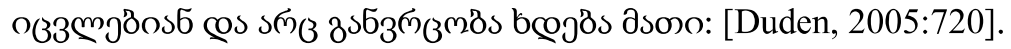


Am Montag hat Donald Trump seinen bisherigen Wahlkampfmanager Bannon, inzwischen politischer Chefstratege im Weißen Haus, auch noch zum ständigen Mitglied im Nationalen Sicherheitsrat ernannt [4].

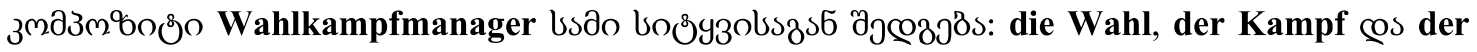

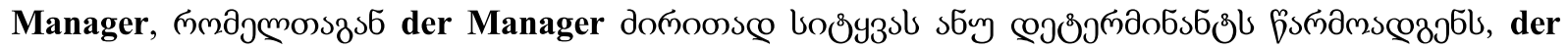

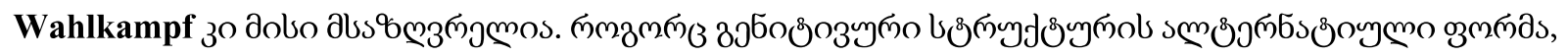

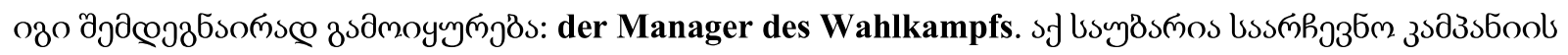
วงธวชวณ์ดว.

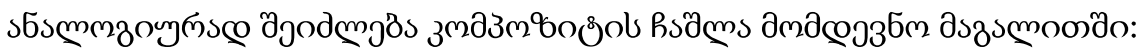

Ein weiteres Gesetz entzieht ihm die Schulaufsicht, reduziert die Verwaltungspositionen und macht die Ernennung von Kabinettsmitgliedern von der Zustimmung des (mehrheitlich republikanischen) Senats abhängig [6].

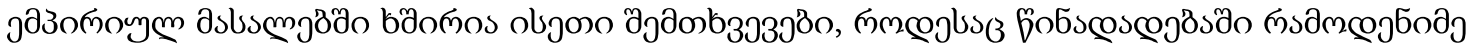

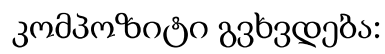

Weil die beiden Fraktionen nur 20 Prozent der Bundestagsabgeordneten stellen, können sie kein Normenkontrollverfahren in Karlsruhe einleiten [7].

Die Bundesregierung hat ganz bewusst Artikel 24 als Grundlage für ihren Mandatstext genommen, weil sie nicht den Verteidigungsfall ausrufen wollte. .[7]

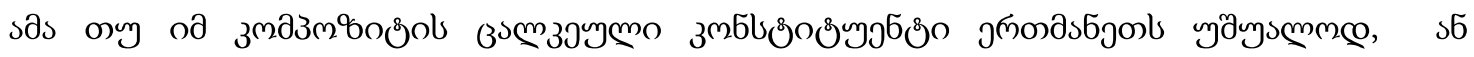

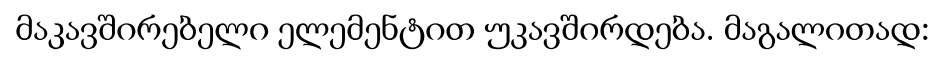

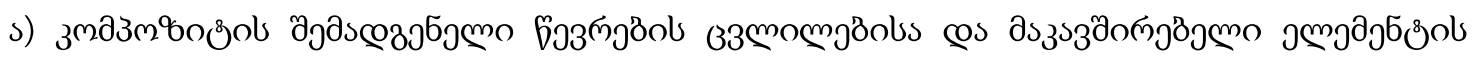
วงกูวฮุ:

Flankiert wurde er dabei von Fabrikarbeitern und sechs seiner sieben Kinder.[7]

Man dürfe das Thema Flüchtlinge allerdings nicht mit dem Terrorismusthema vermischen, sagte Obama.[7]

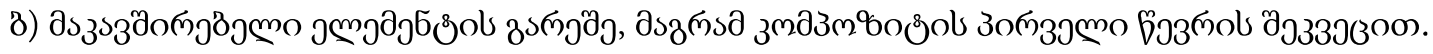

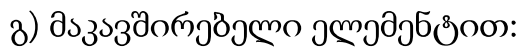

In der Bevölkerung aber genießt Merkel erstaunlich hohe Zustimmungswerte.[6] 
Mit ihrer Mehrheit könnten sie dann die Abstimmungsregeln ändern.[4]

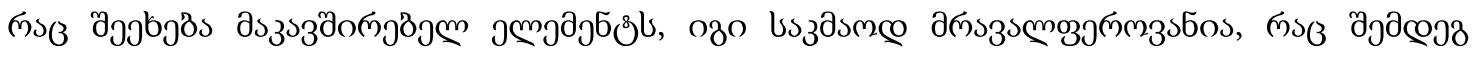

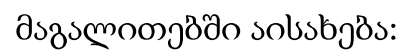

(1) Offen ist noch, wie genau die Zigarettensorten künftig heißen müssen, um die Anforderungen zu erfüllen.[6]

(2) "Weder Sie noch irgendein Bundesbeamter können garantieren, dass syrische Flüchtlinge nicht Teil einer terroristischen Aktivität sein werden", schrieb der Gouverneur.[7]

(3) Den Tiefpunkt in der Zufriedenheitsstatistik hat Merkel nämlich nicht etwa mit der Flüchtlingsfrage erreicht.[6]

(4) Die Vizevorsitzende der Bayern-AfD und Landtagsfraktionschefin Katrin Ebner-Steiner sagte, die Verdächtigen hätten jedes Recht auf Weiterführung ihrer Asylverfahren verspielt.[5]

(5) Die Demokraten, die seit Jahresbeginn die Mehrheit im Repräsentantenhaus haben, bestreiten die Existenz einer solchen „Krise“ und bezeichnen die Mauer als überflüssig und unmoralisch.[5]

(6) Nachdem Markenlogos auf Zigarettenschachteln bereits durch Fotos von Raucherfüßen, Gebissruinen und Kindersärgen verdrängt wurden, sollen demnächst auch alle irgendwie "attraktiv" oder "chic" klingenden Markennamen verschwinden.[4]

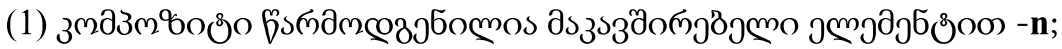

(2) zsдmyјбјв̈умmos -es;

(3) $3^{b} 3 \propto \jmath^{8} \circ 00$-s;

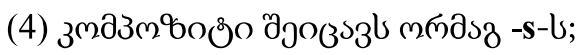

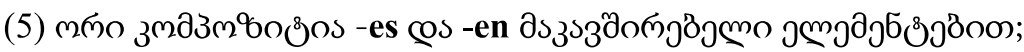

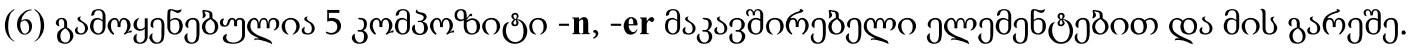

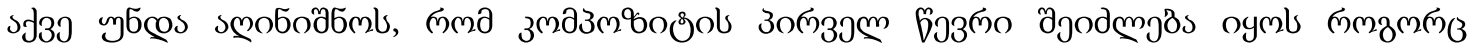

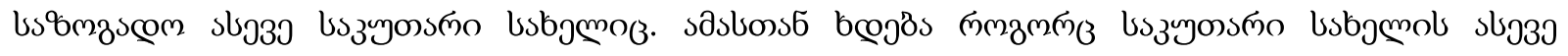

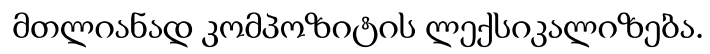

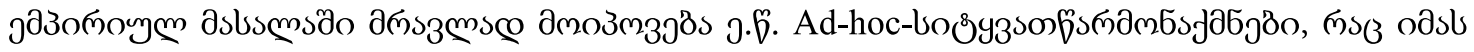

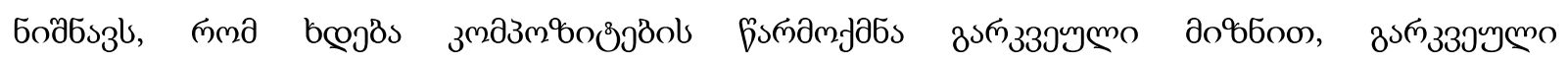




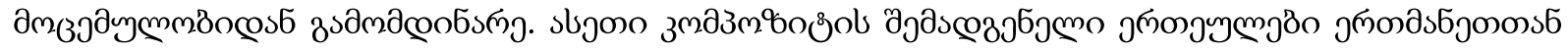

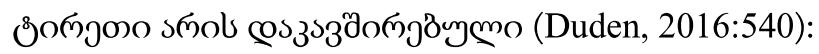

Das von Russland kritisierte EU-Freihandelsabkommen, das an Neujahr in Kraft trat, werde in der finanziell angeschlagenen Ukraine für Arbeitsplätze sorgen, meinte der prowestliche Staatschef.[5]

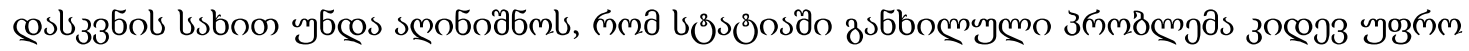

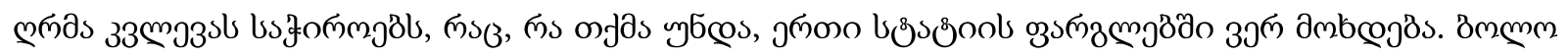

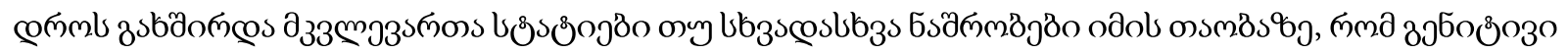

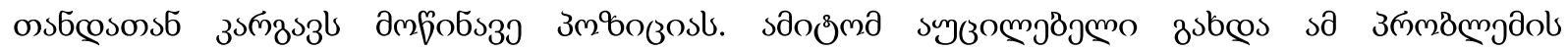

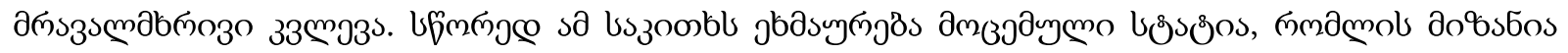

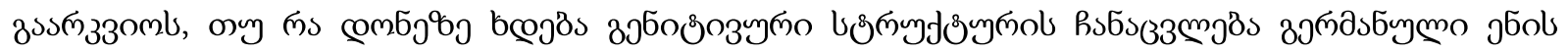

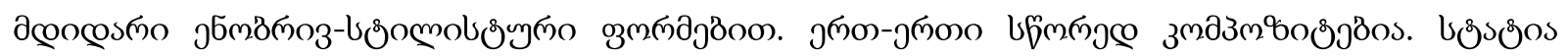

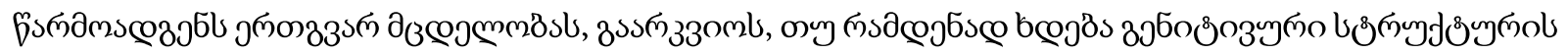

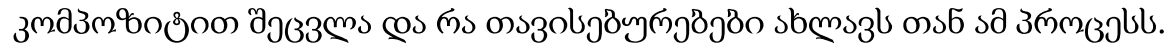

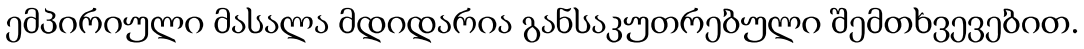

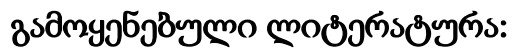

1. DUDEN Die Grammatik, Bd. 4. Bibliographisches Institut \& Brockhaus AG, Mannheim, 2005, S. 720, (Randnummer 1087)

2. DUDEN Die Grammatik, Bibliographisches Institut \& Brockhaus AG, Mannheim, 2016, S. 540.

3. Eichinger L. M., Deutsche Wortbildung Eine Einführung. Gunter Narr Verlag Tübingen. 2000.

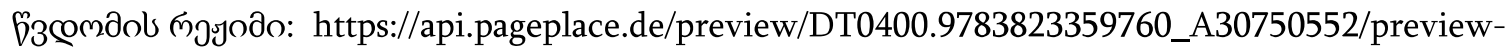

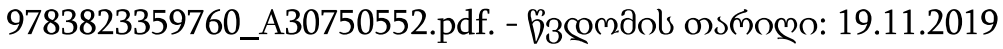

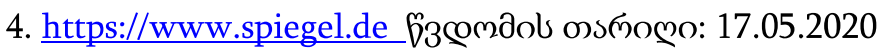

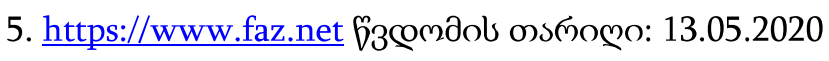

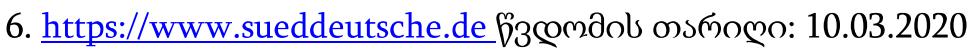

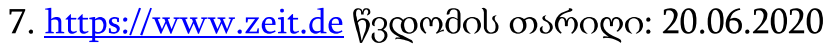


Dali Nikabadze

Akaki Tsereteli State University

\title{
Composite as an Alternative Form of Genetive Structure and its Linguistic-Stylistic Features
}

\begin{abstract}
The article responds to a recent statement on the weakening of the position of the genitive. Numerous studies have dealt with this question. It should be noted, however, that although the genitive actually retreats in relation to the object, it has a fixed position in relation to the attributive function.

The German language is rich in linguistic-stylistic forms that may appear as an alternative to the genitive form. Alternative forms of the genitive, as genitive values, function at different frequencies and degrees. One of their semantic equivalents is composites.

The research is based on the latest entomological literature (P. Eisenberg, P. Galman, L. M. Eichinger, A. Heringer), and the illustrated material is taken from online newspapers (such as Spiegel online, Zeit online, Frankfurter allgemeiner Zeitung, Süddeutsche Zeitung.
\end{abstract}

As for the newspaper genre, comments, reports, interviews were used during the research.

Key words: Genitive, Linguistic-stylistic forms

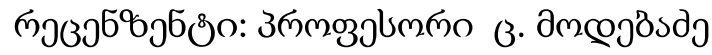

